\title{
A New Algorithm Based on Discrete Fourier Transform to Improve the Lifetime of Underwater Wireless Sensor Networks Communications
}

\author{
Wafa Elmannai, Khaled Elleithy, Ajay Shrestha, Mohamed Alshibli, Reem Alataas
}

\begin{abstract}
Wireless Sensor Networks (WSNs) have become an important means of gathering environmental and physical information from a wide range of areas. WSNs could be used in underground, aboveground and underwater applications. In this paper, we focus on underwater transmission. One of the main limitations of WSNs is the power consumption and short lifetime of the sensors. In this paper, we propose a new solution for underwater Wireless Sensor Networks to overcome the problem that is caused by the ionized nature of seawater. This work presents a methodology to improve the lifetime of WSNs. The wireless sensors have three main functions: sensing, processing and transmitting. The first two factors consume very less power compared to the third. Thus, we need to guarantee the successful transmission of signal with nominal and efficient use of power to improve the lifetime of the sensors. Improving the lifetime of these sensors will improve the experience of the end user, as the information-gathering lifetime of the sensors increases. Based on the results presented in this paper, we can reduce the power consumption, thus improving the lifetime and the signal loss rate.
\end{abstract}

Index Terms - under water communication, WSNs, frequency, energy consumption.

\section{INTRODUCTION}

Underwater sensor networks have a wide variety of applications in real world. They can be used in Ocean Sampling Networks, Pollution, chemical, biological, environmental Monitoring, alerting swimmers about dangerous bacteria levels, disaster prevention, assisted navigation, distributed tactical surveillance, mine reconnaissance, etc [1].

Despite the tremendous potential and use of the technology, there has not been a whole lot of research and development conducted on it yet because there are several challenges.

\section{Manuscript received January 21, 2014.}

W. Elmannai is a Ph.D. candidate in the Department of Computer Science and Engineering at the University of Bridgeport, Bridgeport, CT 06604 USA (e-mail: welmanna@my.bridgeport.edu).

K. Elleithy is the Associate Dean for Graduate Studies in the School of Engineering at the University of Bridgeport, Bridgeport, CT 06604 USA (email: elleithy@bridgeport.edu).

A. Shrestha is a Ph.D. candidate in the Department of Computer Science and Engineering at the University of Bridgeport, Bridgeport, CT 06604 USA (e-mail: shrestha@my.bridgeport.edu).

M. Alshibli is a Ph.D. candidate in the Department of Computer Science and Engineering at the University of Bridgeport, Bridgeport, CT 06604 USA (e-mail: malshibl@my.bridgeport.edu).

R. Alataas is a Ph.D. student in the Department of Computer Science and Engineering at the University of Bridgeport, Bridgeport, CT 06604 USA (email: ralataas@my.bridgeport.edu).
In addition to the well-known resources constraints of the WSNs, Underwater WSNs present even more restrictions that are preventing its proliferation [2, 3]. Unlike the terrestrial networks, Underwater WSNs face multi-path propagation [4] and very long and irregular signal attenuation over long distances. They are also prone to lower bandwidth because lower frequency acoustic waves are used instead of higher frequency electro-magnetic waves to transmit data underwater. They also require more power to operate than their ground counterparts. These and other restrictions have been impeding its development.

Our focus in this paper is to improve the lifetime of WSNs and to increase the distance of signal propagation. Moreover, we propose a framework to alleviate the limitation of wireless communication due to underwater environmental conditions.

\section{A. Underwater Wireless Sensor Networks Deployments (UWSNs)}

Historically underwater monitoring and data collection were performed by recording devices that did not communicate in real time. They simply recorded data and were retrieved at a later point by physically collecting the devices. This would be akin to the use of computers before the advent of the Internet. The disadvantages of the traditional approach included lack of real-time monitoring, inability to interact with control station and limitation on the amount of collected data [5]. Current state-of-art deployments of UWSNs have overcome these shortcomings.

In a typical UWSN as shown in Figure 1.1, a varied range of data including temperature, current, biological (ecosystem productivity), chemical (nutrient fertilization), etc., is collected, processed and finally transmitted by underwater sensors to shore data-collection stations all in real-time [7]. The figure demonstrates the typical deployment of UWSNs. The sensors at the bottom of the ocean floor collect process and relay the data to the surface substation in the moving ship, which in turn transmit the collected information to the onshore sink.

UWSNs deployment differs from terrestrial WSN as shown in Figure 1.2 deployments in a number of ways. The UWSNs are undoubtedly more complex and vulnerable to environmental conditions. The sensors have limited battery power and are extremely difficult, if not impossible to recharge. Due to the fact that acoustic waves are used instead of RF or microwaves, the data transfer rates are very low, 
which is further aggravated by propagation delays. The underwater communication also suffers from multi-path propagation [8] as shown in Figure 1.2.

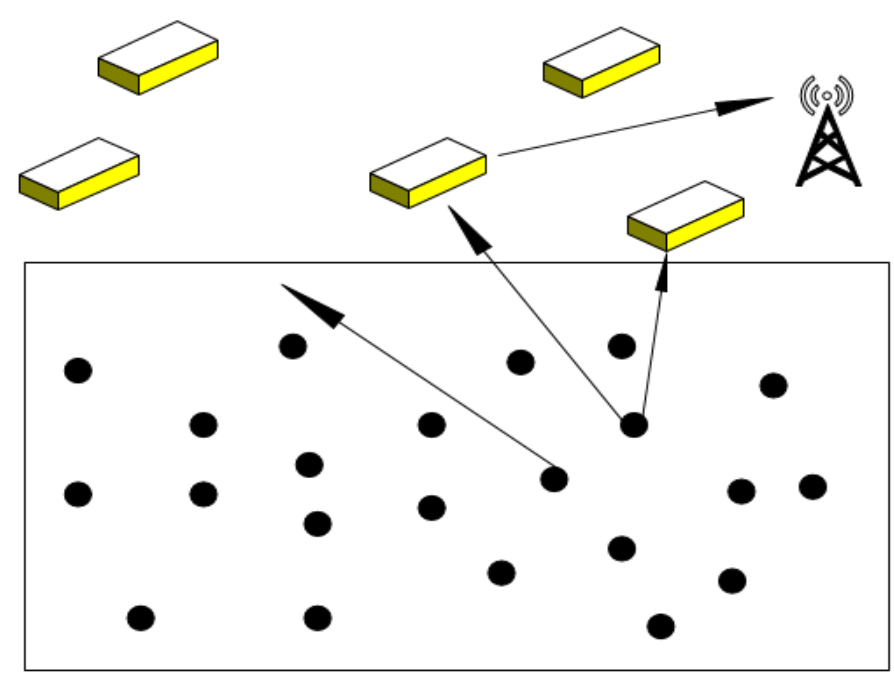

Figure 1.1: An Underwater Acoustic Tested at the University at Buffalo [6]

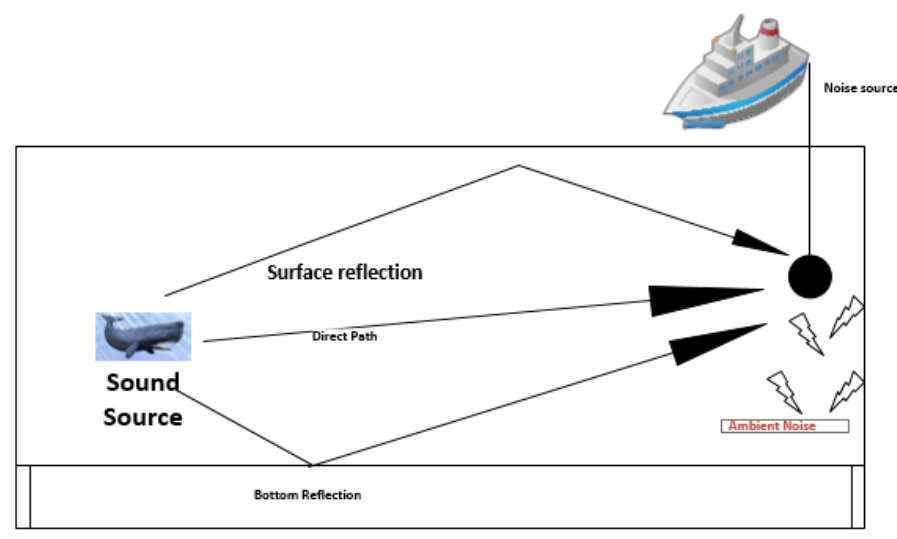

Figure 1.2: Diagram showing underwater Multi-path Propagation

[9]

The differences in the environmental and communication channel characteristics with the terrestrial counterparts, calls for a modification of communication protocols, localization algorithms, error recovery protocols and several other design parameters to fit the needs of UWSNs.

Despite these limitations and challenges, UWSNs have tremendous potential and use to address real world problems. Many governmental and private institutions have identified it as a major research area and have invested in its development.

\section{PROBLEM IDENTIFICATION}

Unlike terrestrial WSNs, electromagnetic waves (RF) are not used in UWSN because the microwave frequency radio waves are absorbed by water. Thus, sound waves are used as a medium to propagate the signal between two points. The biggest challenge for underwater (sea water) communication is that sea water is an ionized medium. Thus, the propagation of sound gets dissipated as the sound travels through the sea water. The sonar frequency has been used as a long term strategy for underwater communications where the communication needs to be done with a trained ear. In this paper, we propose a technique that incorporates underwater communications for sonar frequencies and make it more userfriendly.

\section{RELATED WORK}

During the last decades, developing and improving communications of underwater have become an important area, not only for military issues but also for environmental reasons. Developing safe and efficient way to communicate underwater is important for improving the field of study of underwater world. One of the main tools that are used to keep observation underground is the underwater communication. Sensors are used for this purpose but there are many challenges, e.g., the battery lifetime and the noise effect on the propagated signal. Thus, we focused on how to improve underwater communications. This section provides an overview of the recent developments in this field.

Yusof et. al. [10] presents an in-depth review of underwater communication based on sonar and electromagnetic waves, a comparison of the two systems and a discussion of the environmental impacts of using these waves for underwater communication. As a tradeoff between preserving the underwater environment and the need for underwater communication, it appears that underwater electromagnetic wave communications have the most potential to be the environmentally friendly system of the future. Construction of underwater communication systems may use either sonar wave or electromagnetic wave. The passive sonar communication system simply picks up any sonar waves propagating in the underwater but the active sonar communication system receives as well as emits sonar wave in the underwater. Both passive and active mode use a hydrophone as the device for emitting or receiving sonar waves in the underwater itself. These sonar communication modes are not practical for air-water trans-boundary communication due to significant amplitude attenuation as they cross the air-water interface. Alternatively, electromagnetic wave communication systems are practical for cross-boundary air-water underwater communication almost without any limitation of speed and amplitude attenuation.

Pompili et. al. [11] discuss efficient communication protocols among underwater devices, which are based on acoustic wireless technology for distances over one hundred meters that need to be enabled because of the high attenuation and scattering affecting radio and optical waves, respectively. The unique characteristics of an underwater acoustic channel such as very limited and distance-dependent bandwidth, high propagation delays, and time varying multipath and fading require new efficient and reliable communication protocols to network multiple devices, either static or mobile, potentially over multiple hops. This paper also discusses solutions for medium access control, routing, transport-layer, and crosslayer networking protocols. 
Stojanovic [12] traces back the idea of sending and receiving information under water all the way to the time of Leonardo Da Vinci who is quoted for discovering the possibility to detect a distant ship by listening on a long tube submerged under the sea. In the modern sense of the word, underwater communications began to develop during the Second World War for military purposes. One of the first underwater communication systems was an underwater telephone which was developed in the United States for communicating with submarines. While many problems remain to be solved in the design of high speed acoustic communication systems, recent advances in this area will serve as an encouragement for future work and enable us to remotely explore the underwater world.

Akyildiz et. al. [13] discusses the main challenges of efficient communications in underwater acoustic sensor networks. The paper outlined the peculiarities of the underwater channel with particular reference to networking solutions or monitoring applications of the ocean environment. The objective of this paper is to encourage research efforts to lay down fundamental basis for the development of new advanced communication techniques for efficient underwater communication and networking to enhance ocean monitoring and exploration applications. The paper presents various 2-D and 3-D models that can be used as the basis of future research.

Lloret [14] compares a proposed communication system with other existing systems. Although the proposal supports short communication distances, it provides high data transfer rates. It can be used for precision monitoring in applications such as contaminated ecosystems or for device communications at high depth. The authors have proposed a cheap and efficient way for underwater communications using IEEE 802.11 devices at $2.4 \mathrm{GHz}$ transmission.

Llor et. al. [15] presents the various parameters used for underwater communication. The paper discusses the transmission distance and frequency. Furthermore, the authors investigate the multipath loss. Finally, the paper addresses the modulation and demodulation of the signal for underwater communication.

\section{PROPOSED WORK}

In this paper, we introduce a compression sound algorithm using Fourier series and Morse code in order to improve the wireless underwater sensor communication. Our main purpose of integrating Fourier series and Morse code is to propagate the sound at various frequencies. This will enable the communication to withstand longer multipath propagation. Furthermore, we introduce GUI from text to sound conversion. Figure 2 shows the steps of the algorithm's process.

\section{A. Morse Code:}

Morse code is used to convert the text to on-off tones series, clicks or lights. This can enable the observers and skilled listener to understand the meaning with no specific equipment.
In fact, Morse code is considered as less sensitive to poor signal than the voice. But without a decoding device, it is still a challenge for most researchers. Therefore, in this case the Morse code can be considered as a useful technique to produce speech for distribution of an automatic data to train listeners on voice channels. That can make the first part of our algorithm more efficient in transferring the sound to the data.

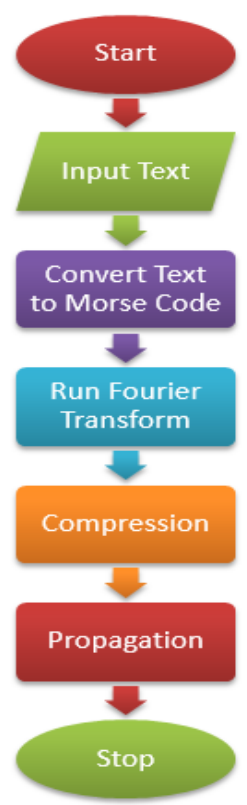

Figure 2: Flowchart of Proposed Model of Wireless Underwater Sensor Communication

\section{B. Discrete Fourier Transform (DFT):}

DFT (Discrete Fourier Transform) or FFT (Fast Fourier Transform) concepts are used interchangeably, and both work with a finite set of values. DFT is used in many mathematical modules where it is fast, since it could be built recursively to reduce the amount of time in execution. DFT or FFT is used to convert from time domain to frequency domain. We can work with the data in the frequency domain easier than the data in the time domain.

\section{Compression Algorithm:}

Analog audio compression allows the efficient storage and transmission of sound data.

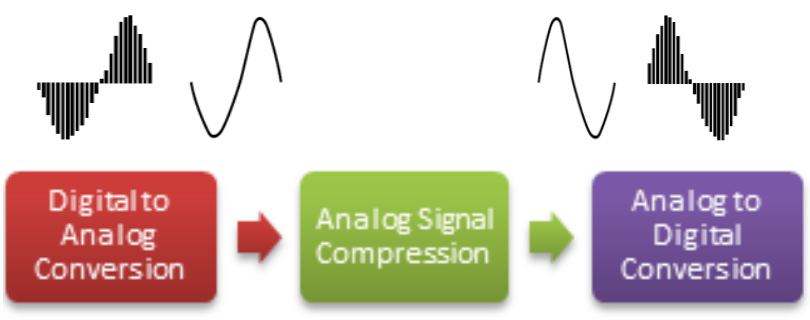

Figure3: Digital to Analog Conversion

Figure 3 shows how the digital audio resulting from running Fourier Transform is converted to analog signals that are ready for compression.

In this implementation, we use the $\mu$-law transformation which is a basic audio compression technique. This 
transformation is a form of logarithmic data compression for an audio data. Due to the fact that we hear logarithmically, sound recorded at higher levels does not require the same resolution as low-level sound. This allows us to disregard the least significant bits in high-level data. This turns out to resemble a logarithmic transformation. The resulting compression forces a 16-bit number to be represented as an 8bit number.

The logarithmic step spacing represents low-amplitude audio samples with greater accuracy than higher-amplitude values. Thus the signal-to-noise ratio of the transformed output is more uniform over the range of amplitudes of the input signal. The $\mu$-law transformation equation is:

$$
y=\left\{\begin{array}{l}
225-\frac{127}{\ln (1+\mu)} \times \ln (1+\mu|x|) \text { for } x \geq 0 \\
127-\frac{127}{\ln (1+\mu)} \times \ln (1+\mu|x|) \text { for } x<0
\end{array}\right.
$$

Where $\mu=255$, and $x$ is the value of the input signal normalized to have a maximum value of 1 .

\section{Simulation SETUP}

We used C\# programming language to implement our approach. The simulation is used to tune the parameters in order to improve the distance in where that signal propagates during transmission. There are some important parameters that can affect the transmission. It can either reduce or increase the power consumption. Hence, if these parameters are not set to the optimal values, the transmitted signal will be lost due to the ionized nature of sea water [15]. Table 1 shows the initial values that can be adjusted using the implemented simulator.

TABLE I

INITIALIZING MORSE CODE AND DFT PARAMETERS

\begin{tabular}{|l|l|}
\multicolumn{1}{|c|}{ Parameters } & \multicolumn{1}{c|}{ Initial Values } \\
\hline Short beep & $0.04 \mathrm{sec}$ \\
\hline Long beep & $0.1 \mathrm{sec}$ \\
\hline Delay & $0.05 \mathrm{sec}$ \\
\hline Frequency & $1000 \mathrm{HZ}$ \\
\hline
\end{tabular}

\section{SimULATION RESUlTS}

Fresh water is ideal for underwater communications because of the absence of ionization. But sea water is ionized, thus it makes the sound dissipate faster. We have used DFT to compress sound signals to a lower frequency to increase the sound propagation distance. This allows us to control the propagation distance while ensuring the security of the information transferred as shown in Figure 4. We do this by modifying the long and short beeps for the Morse code to create new encryption methods to mask the original messages.

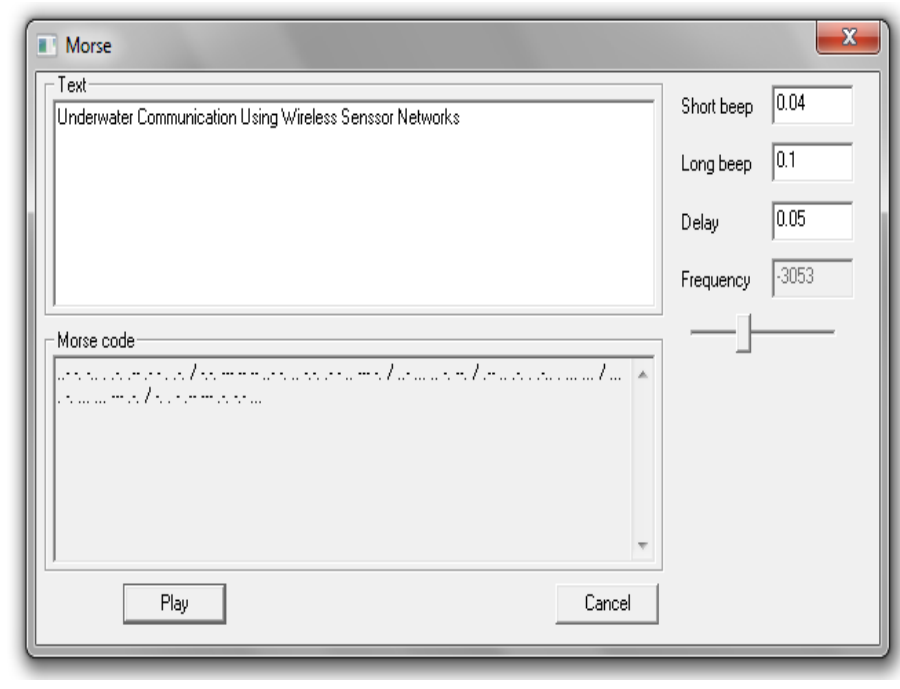

Figure 4: Text to Sound Converter based Morse code

\section{A. Time Average versus Distance Scenario:}

Figure 5 is generated for various sound frequencies over a time average versus distance propagation. It represents a linear format of graphical representation. It shows that increasing sound frequency reduces the time average, but it reduces the propagation distance too. With the reduction in sound frequency, there is an increase in the time average, as well as increase in the propagation distance. From our results, we can conclude that the best solution is to have the maximum propagation distance with the least time average that is close to zero.

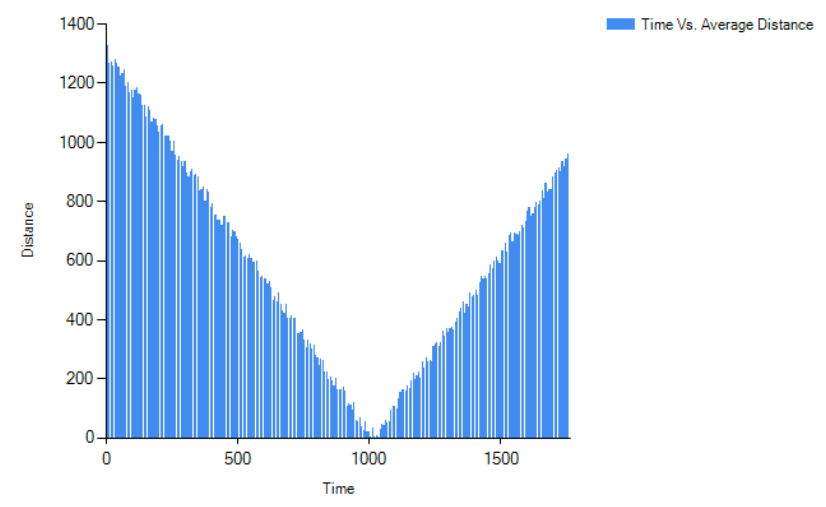

Figure 5: Time Average vs. Distance Propagation Scenario

\section{B. Frequency versus Distance Average Scenario:}

Figure 6 shows the optimal frequency which is required for the best propagation distance that is based on the frequency values. Since our proposed application allows us to change and compress the sound frequency as shown in Figure 4 , we can optimally set the frequency value based on the propagation distance as required. However, the achieved chart is based on the seawater conditions. 


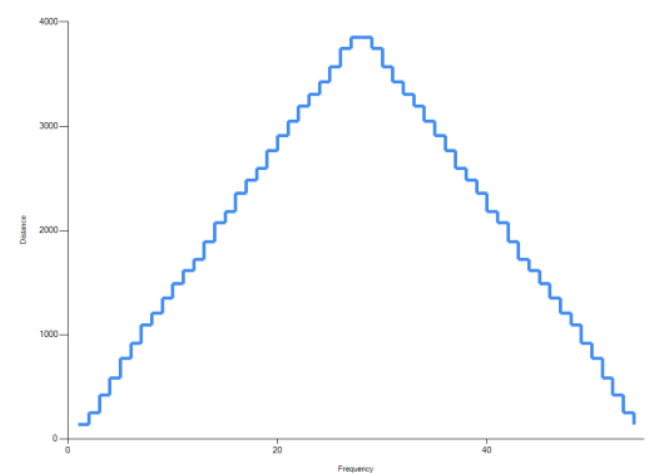

Figure6: Frequency (X-axis) vs. Distance (Y-axis) Average Scenario

\section{CONCLUSIONS}

In this paper, we implemented an algorithm to integrate both Morse code to convert the text to series of tones and DFT to compress the sound signal. This algorithm addresses important problems regarding real-time wireless underwater communication, propagation and ionization.

Propagation distance and ionization of the seawater are some of the most challenging issues of underwater communications. Sound in seawater propagates up to a certain distance and then dissipates.

We presented an efficient algorithm using Morse code and DFT to modify the sound signal based on our requirements. The algorithm tunes the frequency to an optimal value that increases the propagation distance without increasing power consumption.

\section{REFERENCES}

[1] Anupama, K. R., Aparna Sasidharan, and Supriya Vadlamani. "Multi-parameter ocean monitoring system using Underwater Wireless Sensor Networks." In Proceedings of the Fourth IASTED International Conference, vol. 605, no. 224, p. 333. 2008.

[2] Wills, Jack, Wei Ye, and John Heidemann. "Low-power acoustic modem for dense underwater sensor networks." In Proceedings of the 1st ACM international workshop on Underwater networks, pp. 79-85. ACM, 2006.

[3] Akyildiz, Ian F., Dario Pompili, and Tommaso Melodia. "Underwater acoustic sensor networks: research challenges." Ad hoc networks 3, no. 3 (2005): 257-279.

[4] Zhou, Zhong, and Jun-Hong Cui. "Energy efficient multipath communication for time-critical applications in underwater sensor networks." In Proceedings of the 9th
ACM international symposium on Mobile ad hoc networking and computing, pp. 221-230. ACM, 2008.

[5] Underwater Acoustic Sensor Networks (UW-ASN) http://www.ece.gatech.edu/research/labs/bwn/UWASN/in dex.html - Retrieved on March 23rd, 2013.

[6] University of Buffalo (SUNY) - An Underwater Acoustic Testbedhttp://www.eng.buffalo.edu/wnesl/underwater_tes tbed.php.

[7] Autonomous Ocean Sampling Network, Monterey Bay Aquarium Research Institute. http://www.mbari.org/aosn/.

[8] Milica Stojanovic, Massachusetts Institute of Technology, Cambridge, MA 02139. Underwater Acoustic Communications: Design Considerations on the Physical Layer,URL:http://web.mit.edu/millitsa/www/resources/pd fs/garmisch.pdf.pdf

[9] Arnaud Jarrot, Cornel Ioana, Andr' e Quinquis, Laboratory (EA 3876) - ENSIETA, 2RueFrancois Verny, 29806, Brest, FRANCE, Denoising Underwater Signals Propagating ThroughMulti-path Channels URL: http://www.enstabretagne.fr/e3i2/images/E3I2/Publi/publi/JARROT_05a.p df

[10] Yusof, Mohd Ansor Bin, and Shahid Kabir. "Underwater Communication Systems: A Review.", Progress In Electromagnetics Research Symposium Proceedings, Marrakesh, Morocco, Mar. 20-23, 2011.

[11] Pompili, Dario, and I. Akyildiz. "Overview of networking protocols for underwater wireless communications." Communications Magazine, IEEE 47, no. 1 (2009): 97102.

[12] Stojanovic, Milica. "Underwater acoustic communication." Wiley Encyclopedia of Electrical and Electronics Engineering (1999).

[13] Akyildiz, Ian F., Dario Pompili, and Tommaso Melodia. "Challenges for efficient communication in underwater acoustic sensor networks." ACM Sigbed Review 1.2 (2004): 3-8.

[14] Lloret, Jaime, Sandra Sendra, Miguel Ardid, and Joel JPC Rodrigues. "Underwater wireless sensor communications in the $2.4 \mathrm{ghz}$ ism frequency band." Sensors 12, no. 4 (2012): 4237-4264.

[15] Llor, Jesús, and Manuel P. Malumbres. "Underwater Wireless Sensor Networks: how do acoustic propagation models impact the performance of higher-level protocols?." Sensors 12.2(2012):1312-1335. 